\title{
Multiscaling and information content of natural color images
}

\author{
Antonio Turiel* and Néstor Parga ${ }^{\dagger}$ \\ Departamento de Física Teórica, Universidad Autónoma de Madrid, 28049 Madrid, Spain
}

Daniel L. Ruderman

The Salk Institute, 10010 North Torrey Pines Road, La Jolla, California 92037

Thomas W. Cronin ${ }^{\S}$

Department of Biological Sciences, UMBC, Baltimore, Maryland 21228

(Received 2 August 1999; revised manuscript received 31 March 2000)

\begin{abstract}
Naive scale invariance is not a true property of natural images. Natural monochrome images possess a much richer geometrical structure, which is particularly well described in terms of multiscaling relations. This means that the pixels of a given image can be decomposed into sets, the fractal components of the image, with well-defined scaling exponents [Turiel and Parga, Neural Comput. 12, 763 (2000)]. Here it is shown that hyperspectral representations of natural scenes also exhibit multiscaling properties, observing the same kind of behavior. A precise measure of the informational relevance of the fractal components is also given, and it is shown that there are important differences between the intrinsically redundant red-green-blue system and the decorrelated one defined in Ruderman, Cronin, and Chiao [J. Opt. Soc. Am. A 15, 2036 (1998)].

PACS number(s): 42.66.Ne, 87.19.Dd, 47.53.+n, 47.54.+r
\end{abstract}

\section{INTRODUCTION}

The description of the early stages of the visual pathway in mammalians and other animals must be addressed from the knowledge of the properties of the signal that this system is intended to encode: natural images [1-4]. These are very complex objects, and truly random from the point of view of the observer. However, natural images are structured, highly redundant objects, a fact that becomes clear, for instance, when the luminosity changes smoothly over the reflecting surfaces. This redundancy, which should be used as a priori knowledge about the signal, is useful to develop optimal coding strategies, which are learned by the sensory system.

Finding structure in natural scenes is not a trivial problem, and the description of the relevant regularities requires first of all to define the variables where these regularities manifest themselves. One such variable is the contrast, and the analysis of its second-order statistics $[5,6]$ reveals that there is no characteristic scale in the problem [6]. Following this fact, several authors $[3,4,7,8]$ have described natural image statistics in terms of a Gaussian with a $1 / f^{2}$ power spectrum. This was then used to predict the receptive fields of cells in the early visual system. However, it is clear that a Gaussian statistics leaves aside a large amount of qualitatively important structure. This is noticed, for instance, in that once the image is whitened (i.e., the correlations between pairs of pixels are eliminated), the scene can still be recognized thanks to the fact that the borders of the objects are still present [9]. Another piece of evidence against the Gaussian statistics is the presence of long tails in the contrast distribution $[10,11]$.

As it was emphasized in [11-13], a better understanding

\footnotetext{
*Email address: amturiel@delta.ft.uam.es

${ }^{\dagger}$ Author to whom correspondence should be addressed. Email address: parga@delta.ft.uam.es

†Email address: dlr@quake.usc.edu

${ }^{\S}$ Email address: cronin@umbc.edu
}

of the statistics of images should be achieved to define what a natural scene is. This implies the necessity of looking for more regularities. As it was noticed in $[12,14]$, there is further structure that can be detected in the statistics of a quantity related to local changes in contrast. The study of its properties revealed the existence of multiscaling properties in natural scenes: images do not have uniform scale properties, but they can be decomposed in sets of pixels (fractals) such that only those in a given set have similar scale properties. The scaling properties associated with the power spectrum are usually related to the fractal character of images. The new scaling laws observed in monochrome natural images refer to a more detailed structure that reveals that images are not simple fractals (for this notion see, e.g., [15]) but rather multifractal objects (a mathematical concept that was introduced in [16]) which can be split into different fractal sets that transform differently under changes in the scale. The hierarchical structure of the fractal components has been proposed as a natural way of classifying the information content of the visual scenes [13]. Image structure in scale-space has been considered by several authors, although from a different point of view $[17,18]$.

Interestingly enough, these properties can be explained $[14,12]$ by means of a simple model, which obtains the statistics of the contrast gradient at a scale $r$ in terms of an independent multiplicative process applied to the statistics of the contrast gradient at a larger scale $L$. This multiplicative stochastic variable follows a log-Poisson distribution. The events it generates give a statistical description of the way that contrast differences present at the scale $L$ are seen at the finer scale $r$. In particular, a sharp change (modulation) of the contrast gradient is represented in terms of a larger intensity of the multiplicative event. The robustness of the multiscale properties of natural images has been thoroughly tested in a recent work that showed that a wide variety of different image ensembles exhibits a multiplicative process of the logPoisson type [19]. 
The power of the statistical regularities of images detected with these techniques can be appreciated in that they are enough to predict an intrinsic wavelet filter in natural scenes [20]. In fact, the non-Gaussian statistics implied by the logPoisson process uniquely define a wavelet filter that decomposes the image in a set of statistically independent resolution levels, and although it still leaves some spatial dependences, these are extremely short-ranged [20]. This has to be contrasted with the ambiguities that the use of only the power spectrum still leaves in the definition of an optimal filter [3].

It is then relevant to ask whether similar non-Gaussian statistics is also present in color images. In this case, however, one is faced not only with the types of geometrical redundancy mentioned above for monochrome images $[5,6,12]$, but also with chromatic redundancy $[7,21]$. The information conveyed by color images is obviously very redundant, particularly for those spectral channels with the closest wavelengths. One expects that each channel behaves statistically much like a single monochrome channel, with similar geometrical redundancies and strong mutual dependences. Taking as a starting point the usual three-channel red-green-blue (RGB) representation (that we will hereafter call the chromatic system RGB) according to the human sensory receptor classes, Ruderman et al. [21] developed a chromatic system of three new variables (called $l \alpha \beta$ ). As defined, this chromatic system decorrelates the three signals at each point in the image. Thus, these signals define a more compact codification of the RGB images. Moreover, the variables these authors obtain are reminiscent of the chromatic channels of human color vision.

The aim of this work is to explain the chromatic systems both from the geometrical meaning of the fractal components of color images and from the evaluation of the information conveyed by each chromatic channel over the fractal components. We will present the following.

(i) Verification of the log-Poisson multiplicative process for each channel of the two chromatic systems [that is, the standard red-green-blue and the decorrelating one $(l \alpha \beta)]$ [21].

(ii) Performance of a multifractal decomposition of images for the two chromatic systems and a classification of the resulting fractal components, emphasizing the importance and the interpretation of the most relevant of them, the most singular manifold (MSM).

(iii) Determination of the information content and the mutual information among the three components of a given chromatic system, for different sets of pixels (whole image, MSM, and second MSM).

The paper is structured as follows. In Sec. II the instrumental and processing methods used in the elaboration of this work are summarized. The concepts of multiscaling laws and their experimental validations are given in Sec. III. Section IV explains the log-Poisson model which is used to describe the non-Gaussian image statistics. In Sec. V our statistical results are interpreted in geometrical terms, and the decomposition of the images into their fractal components is shown. In addition, the differences between the two chromatic systems are also observed and explained. In Sec. VI a precise measure of the information content and mutual information of the variables are given and interpreted. Finally, the main conclusions are presented in Sec. VII.

\section{METHODS}

The data-gathering methods were as in [21]. Briefly, spectral images were captured using an Electrim EDC-1000TE camera with a resolution of $192 \times 165$ (horizontal $\times$ vertical) eight-bit pixels. Light reaching the CCD array was passed through a variable interference filter with a wavelength range of $400-740 \mathrm{~nm}$ of bandpass typically $15 \mathrm{~nm}$. In each image, 43 successive images were taken of each scene at 7-8 nm intervals from 403 to $719 \mathrm{~nm}$. Each pixel subtended a rectangle of $0.047 \times 0.055$ degrees (horizontal $\times$ vertical). No corrections for optical or CCD-element spatial filtering were made; however, the estimated dark noise was subtracted from each CCD image on a pixel-by-pixel basis. In attempting to select a diversity of typical foliage-dominated scenes, images were collected in several locations around Baltimore, Maryland (temperate woodland) and Brisbane, Australia (sclerophyll forest, subtropical rainforest, and mangrove swamp). Selected scenes contained numerous natural objects, including leaf foliage, bark, rocks, herbs, streams, bare soil, etc. In one corner of each imaged scene small reflectance standards were placed for calibration purposes: a Spectralon $100 \%$ diffuse reflectance material (Labsphere) and a nominally $3 \%$ spectrally flat diffuse reflector (MacBeth).

We collected images of 12 such natural scenes, and further analyzed the central $128 \times 128$ pixel region. Each of the $(128 \times 128 \times 12=196608)$ pixels was converted to three theoretical cone responses as $\Sigma_{\lambda} Q(\lambda) R(\lambda) I(\lambda)$, where $Q(\lambda)$ is the Stockman-MacLeod-Johnson cone fundamental [22] for the given cone type, $R(\lambda)$ is the measured image reflectance data, $I(\lambda)$ is the standard illuminant D65 (which is meant to mimic a daylight spectrum [22,23]), and the sum is over wavelengths represented in the spectrum. Our results depend only very weakly on the choice of illuminant, so long as it is broadband. This procedure provides the cone response data $L(\vec{x}), M(\vec{x})$, and $S(\vec{x})$, proportional to the number of quanta absorbed in an $L, M$, or $S$ cone at spatial location $\vec{x}$ within the image. The raw reflectance data for the 12 images are available via anonymous ftp at ftp://sloan.salk.edu/pub/ruderman/ hyperspectral/.

We will make use of two different chromatic systems of cone response variables to represent each image, the RGB system and the $l \alpha \beta$ system. The RGB system is formed by the raw $L$ (red), $M$ (green), and $S$ (blue) responses and is intended to be an unprocessed representation of the image. The $l \alpha \beta$ system is formed by the variables obtained in [21], which is defined as follows:

$$
\begin{gathered}
l=\frac{1}{\sqrt{3}}(\ln L+\ln M+\ln S)-l_{0}, \\
\alpha=\frac{1}{\sqrt{6}}(\ln L+\ln M-2 \ln S)-\alpha_{0}, \\
\beta=\frac{1}{\sqrt{2}}(\ln L-\ln M)-\beta_{0},
\end{gathered}
$$

where $l_{0}, \alpha_{0}$, and $\beta_{0}$ are appropriate constants verifying that the average of each variable over each image is equal to zero. These variables are decorrelated, that is, the average of the 
product of any two different variables vanishes (see [21]). This decorrelation property is a weak kind of independence (if the variables are independent, then they are decorrelated).

\section{STATISTICS OF IMAGES: MULTISCALING}

It is believed that natural images behave like "fractal" objects: they do not possess a scale of reference and they are self-similar [6], each small portion of them behaving in the same way as the whole image (in a statistical sense). However, the kind of self-similar behavior shown by the power spectrum is insufficient to provide a detailed description of the local structure of natural scenes [10-13]. This is because it assigns the same scaling exponent to every image pixel. To obtain a better description of the image, it is necessary to define a variable with a local scope, able to detect its local features. The hope is that a variable like this could assign distinct self-similar behaviors to different pixels, which in turn could be used to detect and classify its local features. Examples of this approach can be found in $[12,13,19]$, where dealing with gray-scale images a whole hierarchy of image features, from sharp edges to textures, has been put in correspondence with local scaling exponents.

In this work the approach is extended to color images. The existence of a hierarchy is explored and explicitly checked for all the components of the chromatic systems presented in the preceding section. In analogy with the variables defined in $[12,13]$, given any of the chromatic components presented in Sec. II, the edge content (EC) of this component at the point $\vec{x}$ and at the scale $r, \epsilon_{r}(\vec{x})$, is defined as

$$
\epsilon_{r}(\vec{x})=\frac{1}{r^{2}} \int_{B_{r}(\vec{x})} d \vec{x}^{\prime}|\nabla C|\left(\vec{x}^{\prime}\right)
$$

where $C(\vec{x})$ denotes the selected chromatic component (e.g., $\mathrm{R}, \mathrm{G}, \mathrm{B}, l, \alpha$, or $\beta$ ). The bidimensional integral is defined over $B_{r}(\vec{x})$, which represents a square of linear size $r$ centered at $\vec{x}$. We will often use one-dimensional surrogates of the EC, which are statistically less demanding. These are defined as integrals along a direction given by a vector $\vec{r}$ of length $r$ :

$$
\epsilon_{r}^{\vec{r}}(\vec{x})=\frac{1}{r} \int_{-r / 2}^{r / 2} d s\left|\frac{\partial C}{\partial s}\left(\vec{x}+s \frac{\vec{r}}{r}\right)\right|
$$

As noted before, these variables compute the average over a scale $r$ of a quantity that compares two neighboring points. It is then clear that even its marginal distribution contains information about the local structure of the image. This is not the case for the more usual average over the same scale of the chromatic components themselves.

We now introduce the important concepts of selfsimilarity (SS) and extended self-similarity (ESS) (see [12]). Given a random variable $\epsilon_{r}$ defined on a local area of size $r$, we would say that this variable has SS if its statistical moments of order $p$ obey a power law with exponent $\tau_{p}$ :

$$
\left\langle\epsilon_{r}^{p}\right\rangle=\alpha_{p} r^{\tau_{p}}
$$

where $\alpha_{p}$ is a geometrical factor. The angular brackets $\langle\cdots\rangle$ represent the average over all the points $\vec{x}$ belonging to each image for all the images in the ensemble considered. Since $\tau_{p}$ is an arbitrary function of $p$, this is a more general type of scaling than the one observed in the power spectrum. The simplest possible system exhibiting SS is that in which $\tau_{p} \propto p$. In that case, the dependence on the scale parameter $r$ is trivial: it simply implies that the moments of the normalized variable $\epsilon_{r} /\left\langle\epsilon_{r}\right\rangle$ do not depend on $r$. The most interesting cases are those in which $\tau_{p} \neq \tau_{1} p$, and this deviation is known as anomalous scaling.

The concept of ESS requires that the moments verify a weaker identity:

$$
\left\langle\epsilon_{r}^{p}\right\rangle=A(p, q)\left\langle\epsilon_{r}^{q}\right\rangle^{\rho(p, q)} .
$$

Any moment of order $q$ could be used (provided $\tau_{q} \neq 0$ if SS holds); we will always use the moment $q=2$. If $\epsilon_{r}$ has SS, it also has ESS, and the relation between the exponents $\tau_{p}$ and $\rho(p, 2)$ is

$$
\rho(p, 2)=\frac{\tau_{p}}{\tau_{2}} .
$$

We can now verify if SS and ESS hold for color-natural images using the dataset presented in Sec. II. For this purpose we have used the variables $\epsilon_{r}(\vec{x})$ [Eq. (3)] taking $\vec{r}$ in both the horizontal and the vertical directions. The numerical analysis was done over the six EC variables built on the six chromatic components RGB and $l \alpha \beta$. The scale $r$ was taken small compared with the total size of the image $(r \leqslant 64$ pixels) and $p$ was taken up to $p=10$. It is remarkable that both SS and ESS hold for all of these cases. The test for ESS is presented for the horizontal EC of the chromatic components RGB in Fig. 1 and for the chromatic components $l \alpha \beta$ in Fig. 2. The ESS exponents $\rho(p, 2)$ are shown in Fig. 3, again for the three components of the two chromatic systems.

The exponents $\tau_{p}$ do not behave linearly with $p$. This proves that natural scenes possess nontrivial scaling laws, and that more complex regularities are present in the image ensemble. The next section describes a model that predicts the correct exponents and consequently contains those regularities. After that, the statistical approach followed here will be related with a local approach, this will allow us to predict geometrical properties of natural images from the knowledge of $\tau_{p}$.

\section{MULTIPLICATIVE PROCESSES: LOG-POISSON MODEL}

The data presented in the preceding section show that ESS holds for the six chromatic components discussed in this work. We show here that a very simple model, based on a log-Poisson multiplicative process [24-26,12], is able to fit these data. The existence of such a process means that the $\mathrm{EC}$ at a scale $r$ is obtained from the EC at a greater scale $L$ by multiplying it by a random variable $\alpha_{r L}$ :

$$
\epsilon_{r}=\alpha_{r L} \epsilon_{L}
$$



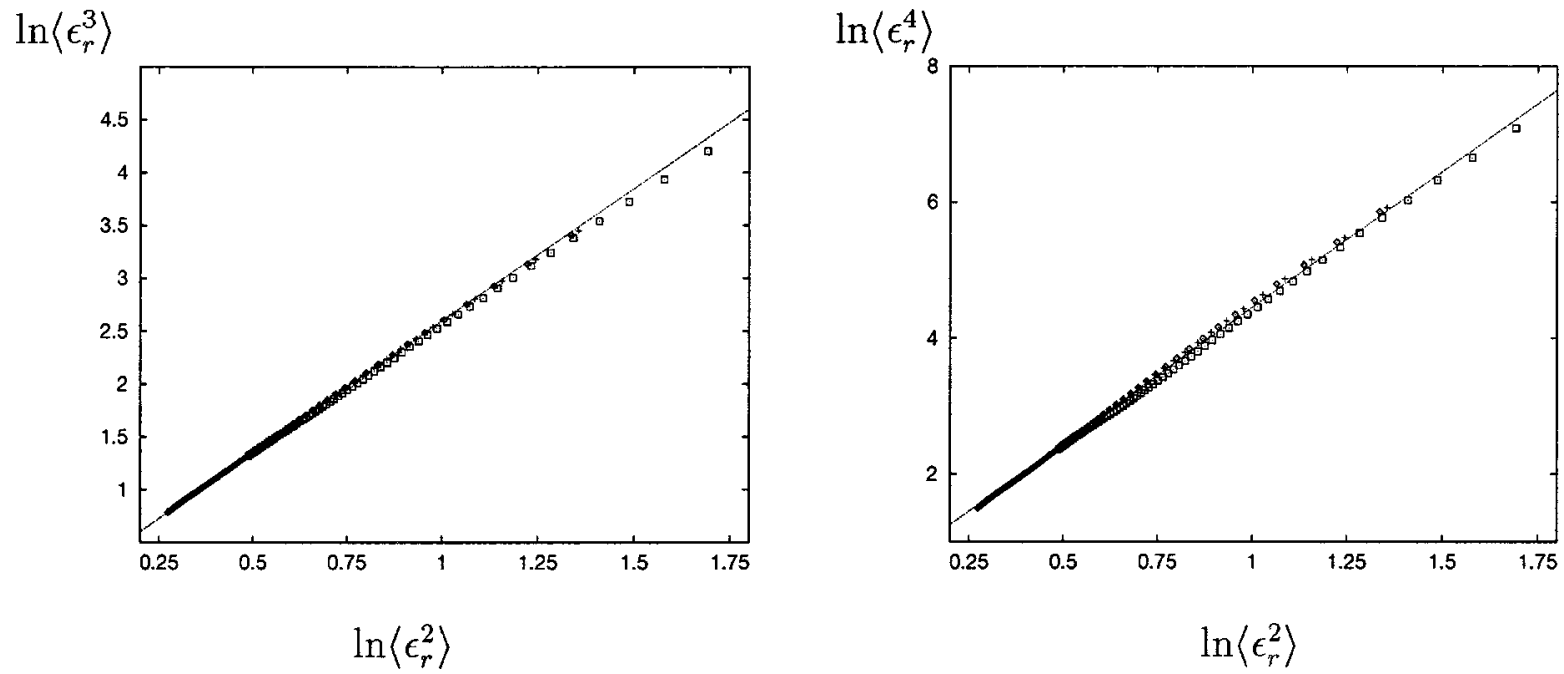

FIG. 1. Verification of ESS for the third- and fourth-order moments of the horizontal EC in the RGB chromatic system (diamonds, red; crosses, green; boxes, blue). The best linear fits are also represented. Each data point corresponds to a fixed value of $r$, from 4 to 64 pixels. Although not shown here, the vertical EC gives an equally good fit.

where $\alpha_{r L}$ is independent of $\epsilon_{L}$. The random factors $\alpha_{r L}$ define the multiplicative process, and for any intermediate scale $r^{\prime}, r<r^{\prime}<L$, the following relation must hold:

$$
\alpha_{r L}=\alpha_{r r^{\prime}} \alpha_{r^{\prime} L}
$$

This implies that the process can be infinitely split into many intermediate stages, and it is thus said to be infinitely divisible. The factor $\alpha_{r L}$ takes account of the consecutive transitions of the EC from a large scale in the image to smaller ones. Knowing the process and the probability distribution of the EC at the largest scale $L$, the probability distribution of the EC at any other scale $r<L$ can be computed.

Under an infinitesimal change in the scale (when the EC at scale $r$ is generated from the EC at scale $r+d r)$, the log-Poisson model is a binomial distribution with one event infinitely less probable than the other. The most probable

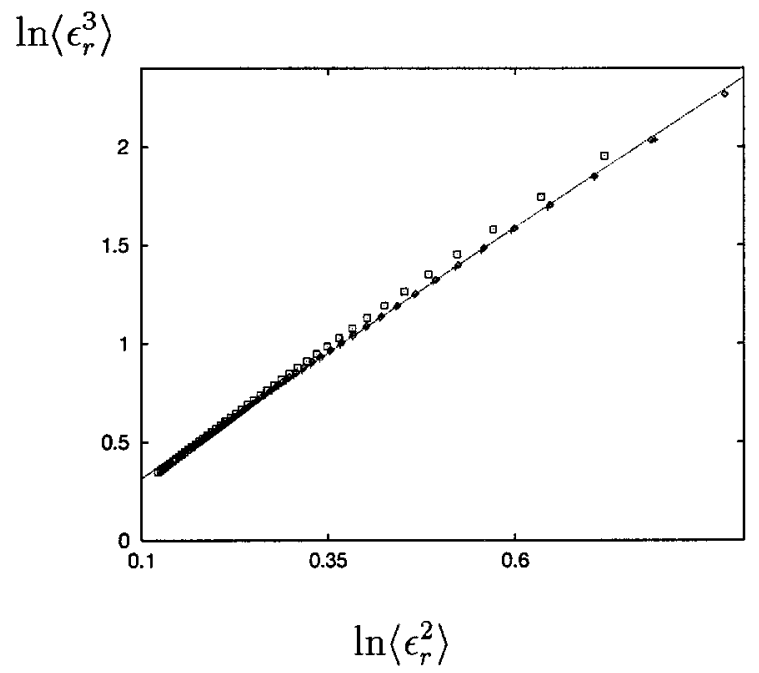

event corresponds to smooth transitions in the contrast (e.g., the surface of an object), whereas the infinitesimally rare event indicates a sharp transition (e.g., an edge). The sharpness of the transition is observed as a finite change of the value of $\alpha_{r L}$ under an infinitesimal change in the scale $r$; we will characterize this finite change by means of a modulation parameter, $0 \leqslant \beta \leqslant 1$, which measures the fraction of the value of $\alpha_{r L}$ which remains after that transition. More precisely, the $\alpha_{r L}$ 's are obtained by

$\alpha_{r, r+d r}= \begin{cases}1+\Delta \frac{d r}{r} & \text { with probability } 1-\left[d-D_{\infty}\right] \frac{d r}{r} \\ \beta\left(1+\Delta \frac{d r}{r}\right) & \text { with probability }\left[d-D_{\infty}\right] \frac{d r}{r},\end{cases}$

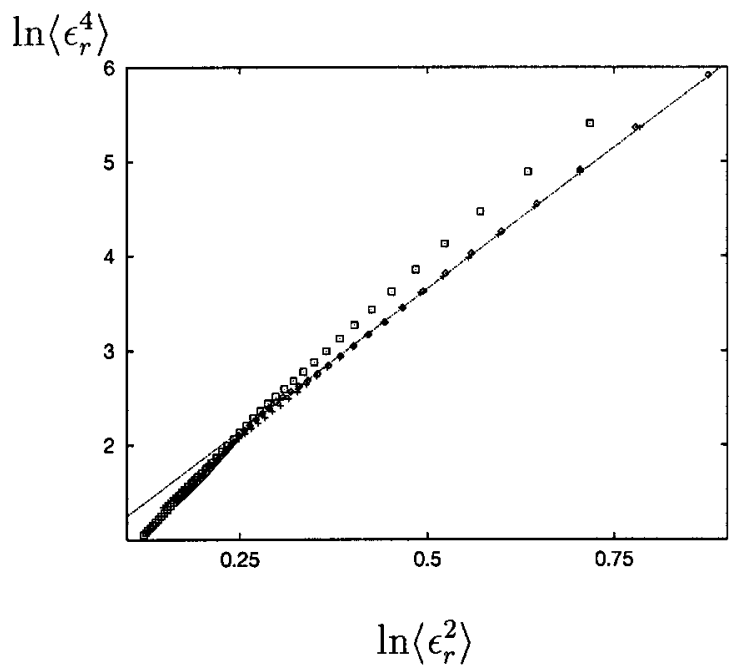

FIG. 2. Verification of ESS for the third- and fourth-order moments of the horizontal EC in the $l \alpha \beta$ system. (Diamonds, $l$; crosses, $\alpha$; boxes, $\beta$.) The best linear fits are also represented. It is observed that the $\beta$ component (boxes) deviates significantly from the others, probably because this component lacks numerical accuracy (it is given by the difference between the color channels with the nearest wavelengths). Each data point corresponds to a fixed value of $r$, from 4 to 64 pixels. Although not shown here, the vertical EC gives an equally good fit. 


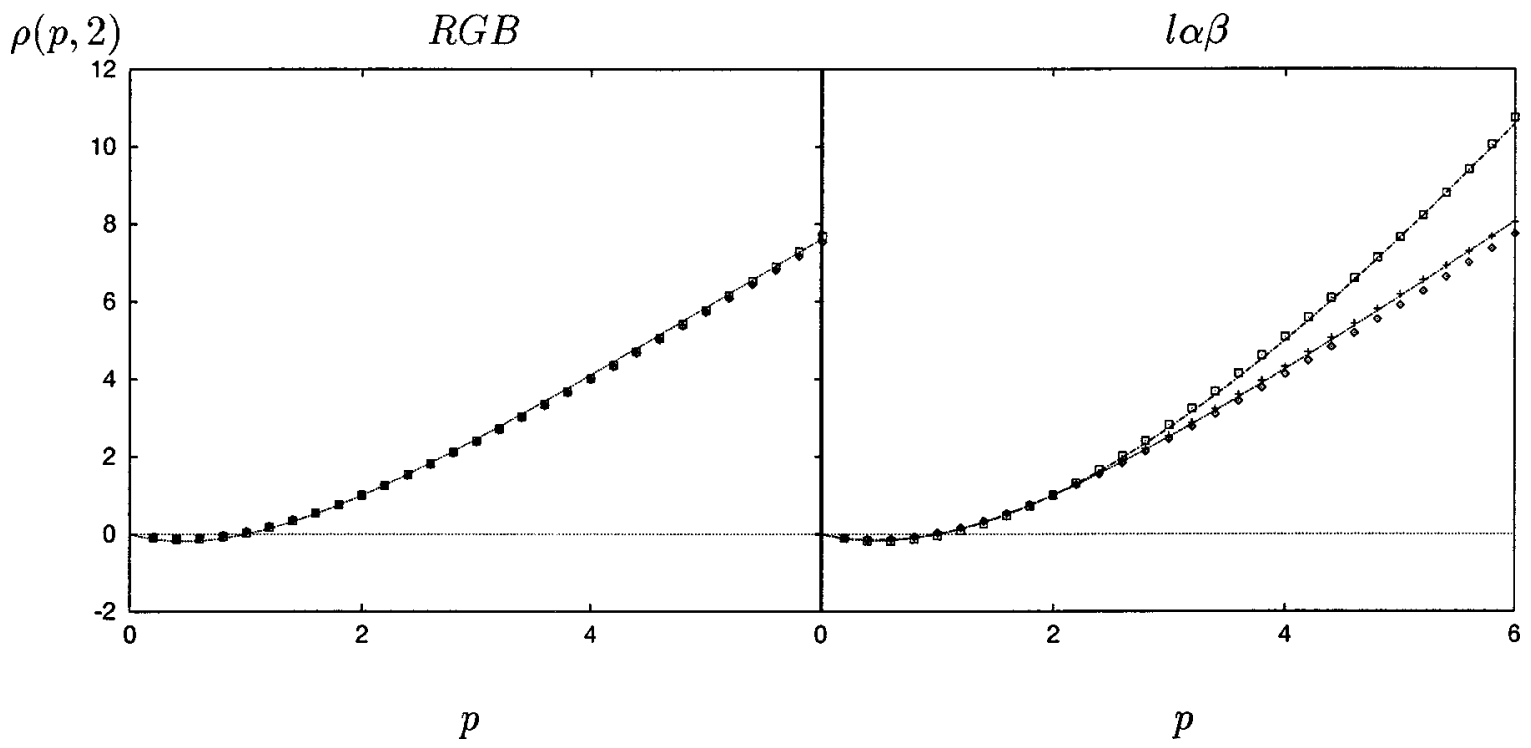

FIG. 3. ESS coefficients $\rho(p, 2)$ for the RGB (left) and the $l \alpha \beta$ (right) chromatic systems, and the horizontal EC (symbols as in Figs. 1 and 2). The comparison with the log-Poisson model prediction (as described in Sec. IV) is also represented for each component. RGB system: $\beta_{R}=0.45, \beta_{G}=0.45$, and $\beta_{B}=0.46 ; l \alpha \beta$ system: $\beta_{l}=0.50, \beta_{\alpha}=0.50$, and $\beta_{\beta}=0.73$.

where the parameters $\Delta$ and $D_{\infty}$ can be expressed in terms of the SS exponent $\tau_{2}$ and the modulation parameter $\beta$ by (for details, see [13])

$$
\begin{gathered}
\Delta=-\frac{\tau_{2}}{1-\beta}, \\
d-D_{\infty}=-\frac{\tau_{2}}{(1-\beta)^{2}} .
\end{gathered}
$$

Here $d$ is the dimensionality of the system; $d=2$ for our images. When a noninfinitesimal change in scale is considered, this formula leads to a log-Poisson distribution for the multiplicative process $\alpha_{r L}$. The probability distribution of $\alpha_{r L}, \rho_{\alpha_{r L}}(\alpha)$, is given by

$$
\rho_{\alpha_{r L}}(\alpha)=e^{-s_{r L}} \sum_{n=0}^{\infty} \frac{s_{r L}^{n}}{n !} \delta\left(\alpha-\beta^{n}\left[\frac{L}{r}\right]^{\Delta}\right),
$$

where $s_{r L}=\left(d-D_{\infty}\right) \ln (L / r)$ is the average number of modulations between the two scales. Notice that this distribution depends only on the ratio between the two scales. This model has been used previously to describe turbulent flows [27] and gray-scale natural images $[12,13]$.

It is now easy to compute the SS exponents $\tau_{p}$ : from Eqs. (7) and Eqs. (5) it follows that $\left\langle\alpha_{r L}^{p}\right\rangle=[r / L]^{\tau_{p}}$. The moments of $\alpha_{r L}$ can be computed using Eq. (11) as

$$
\begin{aligned}
\left\langle\alpha_{r L}^{p}\right\rangle & =e^{-s_{r L}} \sum_{n=0}^{\infty} \frac{s_{r L}^{n}}{n !} \beta^{n p}\left[\frac{L}{r}\right]^{\Delta p} \\
& =\left[\frac{L}{r}\right]^{\Delta p-\left(d-D_{\infty}\right)\left(1-\beta^{p}\right)}
\end{aligned}
$$

using $e^{-s_{r L}}=[L / r]^{d-D_{\infty}}$. It follows that $\tau_{p}=-\Delta p+(d$ $\left.-D_{\infty}\right)\left(1-\beta^{p}\right)$. The ESS exponents are then calculated using $\rho(p, 2)=\tau_{p} / \tau_{2}$ and the definitions of $\Delta$ and $D_{\infty}$, Eq. (10); they are given by

$$
\rho(p, 2)=\frac{p}{1-\beta}-\frac{1-\beta^{p}}{(1-\beta)^{2}} .
$$

It is remarkable that the ESS exponents depend only on the modulation parameter $\beta$. Besides, using Eq. (6) one sees that the set of SS exponents $\tau_{p}$ 's can be computed with only two parameters (namely, $\beta$ and $\tau_{2}$ ). The validity of the model can be tested by fitting the ESS exponents $\rho(p, 2)$ with Eq. (13). The agreement between model and data for the two chromatic systems is shown in Fig. 3.

To conclude this section, let us notice that Eq. (7) allows us to compute the distribution of $\alpha_{r L}$ from the distributions of the EC's at the scales $L$ and $r$, by deconvolution. (Notice that this deconvolution problem is numerically ill-posed. As a consequence, the distribution of $\alpha_{r L}$ so obtained is less precise than the one inferred by fitting the moments of the EC.) Two examples of these distributions are shown in Fig. 4, together with the log-Poisson distribution [Eq. (11)]. Notice that a log-Poisson distribution becomes eventually lognormal. The reason is that the infinitely divisible character of $\alpha_{r L}$, expressed in Eq. (8), implies that $\ln \alpha_{r L}$ is the sum of an infinite number of independent random variables. Provided that the dispersion of that sum is small compared with its mean, this process will get closer to normal. This is not seen in Fig. 4 because at the two considered scales the average number of transitions is rather small. This is a manifestation of the fact that natural images present far-from-Gaussian behavior.

\section{GEOMETRY OF CHROMATIC COMPONENTS: THE MULTIFRACTAL REPRESENTATION}

The anomalous scaling laws for the moments of the chromatic EC's can be explained on the basis of local anomalous 

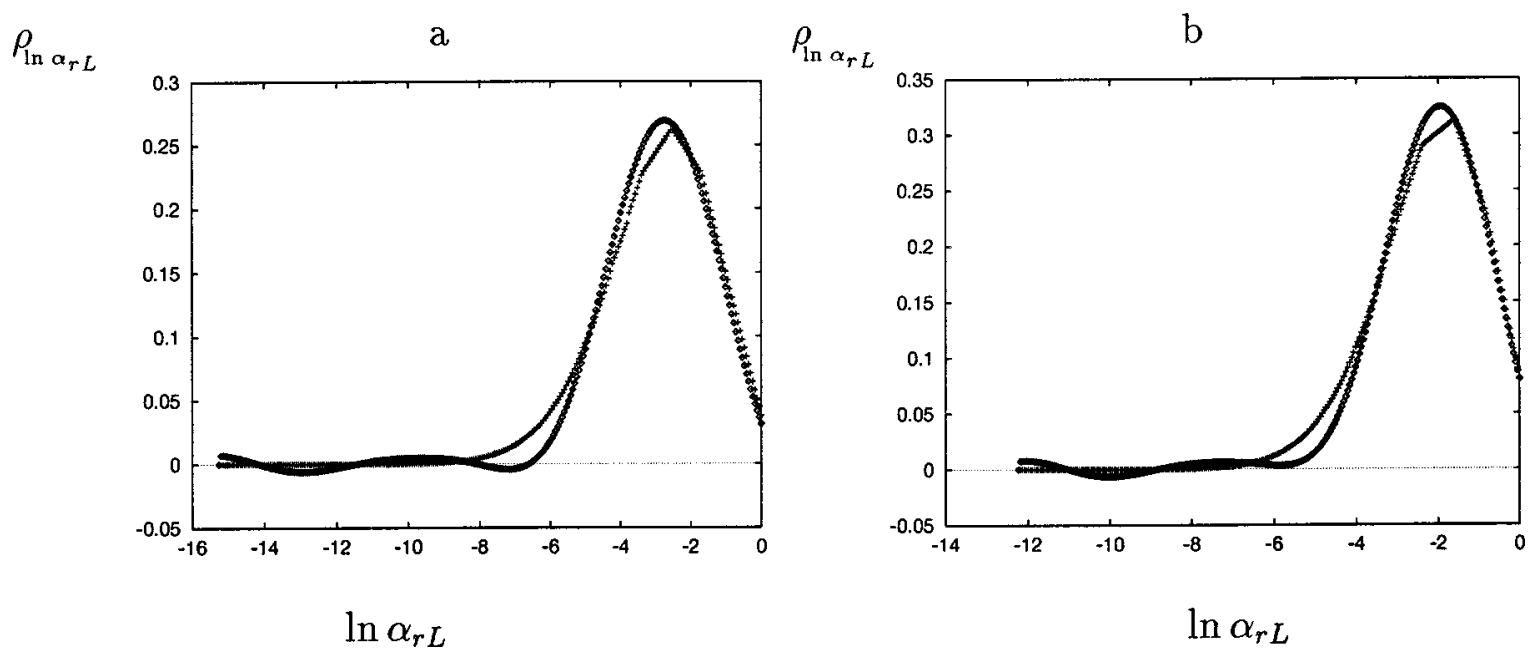

FIG. 4. Experimental distributions (diamonds) of $\ln \alpha_{r L}$ for (a) the horizontal red EC and (b) the horizontal $l$ EC; $r=4$ pixels, $L=64$ pixels. Both distributions are far from Gaussian, but very close to a Poisson distribution with the appropriate parameters (crosses). The average number of transitions ( $\beta$ modulations) for both processes is the same: $s=\left(d-D_{\infty}\right) \ln (L / r)=2.77$. This number is rather small to yield a Gaussian behavior.

scaling exponents. We define the edge measure (EM) for a given chromatic component of a square of side $r$ centered around a point $\vec{x}, \mu\left(B_{r}(\vec{x})\right)$, as

$$
\mu\left(B_{r}(\vec{x})\right)=r^{2} \epsilon_{r}(\vec{x}),
$$

so the EC is just a comparison between the EM of a square and its standard area $r^{2}$. The convenience of the definition of the EM with respect to that of the EC is given by the fact that the EM is additive: if, for instance, a square is split in several pieces, the EM of the square is the sum of the EM's of its parts. It is natural to ask whether the EM of a square shows a local power-law scaling as

$$
\mu\left(B_{r}(\vec{x})\right)=\alpha(\vec{x}) r^{h(\vec{x})+2}
$$

where $h(\vec{x})$ is the local scaling exponent of a chromatic component at the point $\vec{x}$. A negative value of $h(\vec{x})$ means that the gradient of the contrast possesses a singularity at $\vec{x}$, and its numerical value characterizes the type of the singularity. If $h(\vec{x})>0$, the gradient is continuous at $\vec{x}$; if $h(\vec{x})>1$, it is differentiable; etc. The exponent $h(\vec{x})$ will be referred to as the singularity exponent at $\vec{x}$ (understanding that positive values are a measure of the degree of "regularity"). A measure verifying Eq. (15) is said to be multifractal. The reason for this name is that any image with a multifractal measure can be decomposed in sets $F_{h}$ which will be observed to have nontrivial fractal dimensions. For each chromatic component, $F_{h}$ is the set of points with the same exponent $h$, and will be referred to as the fractal component of exponent $h$. The fractal dimension of each set will be denoted as $D(h)$ and this function is called the singularity spectrum [16] of the multifractal.

The characterization of a multifractal system by means of its fractal components has been shown to be a powerful tool in multiscaling systems $[30,13]$. In fact, there exists a strong link between the statistical property of SS and the geometri- cal one of multifractality. It is a well-known fact [16] that $D(h)$ is the Legendre transform of $\tau_{p}$ and vice versa, that is,

$$
D(h)=\inf _{p}\left\{p h+d-\tau_{p}\right\}
$$

(inf stands for the infimum; here $d=2$ ). This allows not only the computation of the singularity spectrum from the statistical data, but also the determination of the range of observed local singularities $h$. In the log-Poisson model, the whole singularity spectrum is determined by only two parameters. For instance, these can be chosen as $\beta$ and $\tau_{2}$, and so it reads $[27,13]$

$$
D(h)=d+\frac{\tau_{2}}{(1-\beta)^{2}}-\frac{h-\frac{\tau_{2}}{1-\beta}}{\ln \beta}\left[1-\ln \left(\frac{h-\frac{\tau_{2}}{1-\beta}}{\frac{\tau_{2} \ln \beta}{(1-\beta)^{2}}}\right)\right] .
$$

It is remarkable that according to Eq. (17), the fractal dimensions of the components $F_{h}$ in a log-Poisson SS are nontrivial; moreover, the observed values have a continuous range. On the other hand, the singularity spectrum can be more compactly expressed in terms of the free parameters $\Delta$ and $D_{\infty}$ defined in Eq. (10) as

$$
D(h)=D_{\infty}+\left(d-D_{\infty}\right) w(h)[1-\ln w(h)],
$$

where $\omega(h)=-(h+\Delta) /\left\{\left(d-D_{\infty}\right) \ln \left[1-\left(\Delta / d-D_{\infty}\right)\right]\right\}$, a linear function of $h$. The fractal component with smallest exponent, $F_{\infty}$, is called the most singular manifold (MSM). It turns out that $-\Delta$ is the exponent characterizing the MSM $\left(F_{\infty} \equiv F_{-\Delta}\right)$ and $D_{\infty} \equiv D(-\Delta)$ is its dimension [12]. For example, for a log-Poisson model with $\beta=0.5$ and $\tau_{2}=$ -0.25 (which are close to the values experimentally observed in our data set) one obtains that $\Delta=0.5, D_{\infty}=1.0$.

Let us remark that $\Delta$ and $D_{\infty}$ can be chosen as the two free parameters of the model [see Eq. (10)]. This means that the fractal dimension and the singularity exponent of the 


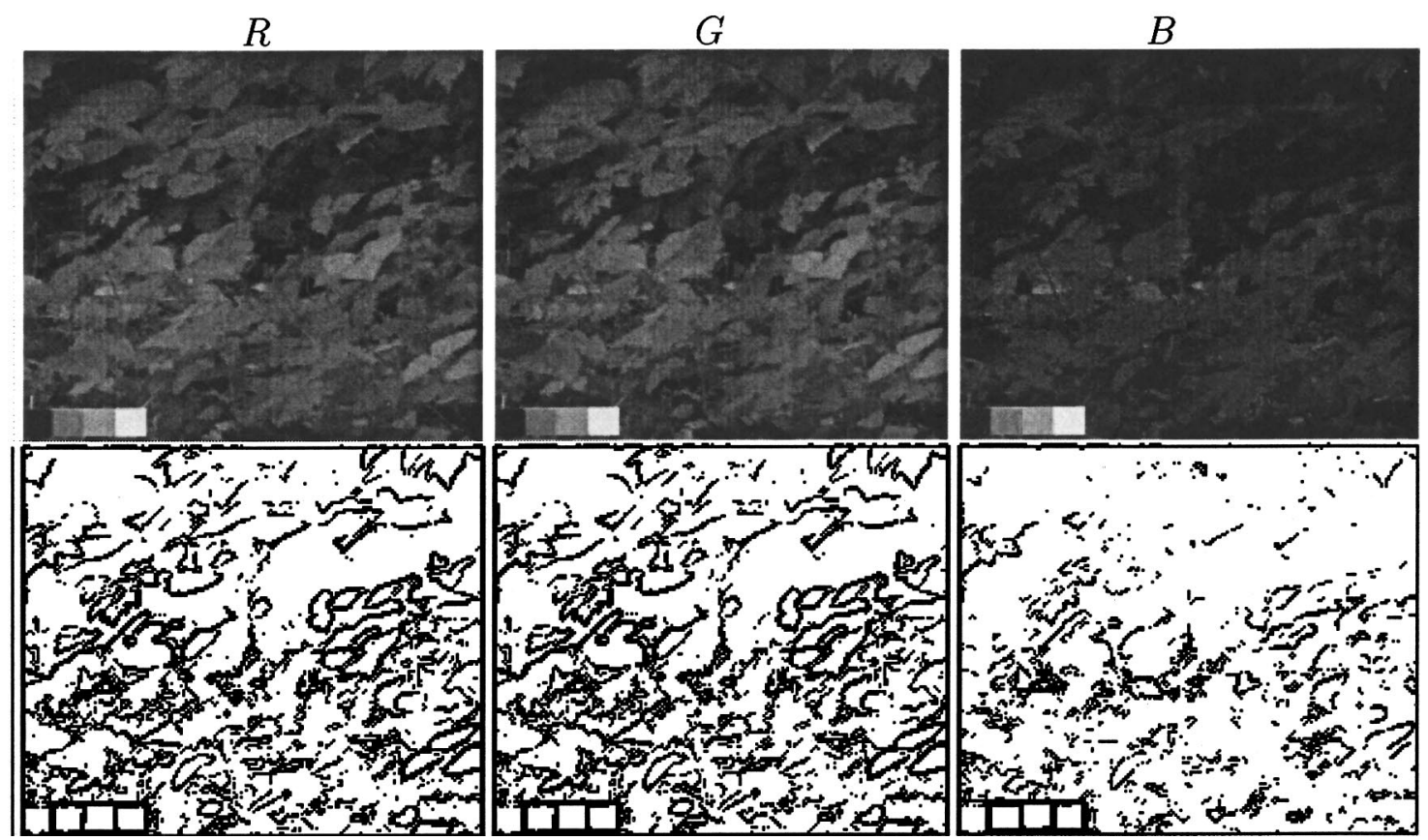

FIG. 5. Gray-level representations of the RGB system (top) and the associated MSM's (bottom) on Park2 image. The MSM's were taken as the sets of points having exponent $h=-0.5 \pm 0.1$. The blue component has significantly less contrast than the others, which makes it difficult or even impossible to detect some of its edges. The MSM's of all members in the RGB system are, however, very similar.

MSM determine all the multifractal properties. This fact stresses the relevance of this particular set of pixels and suggests that it may contain enough information to reconstruct the whole image [28].

There are several ways of computing explicitly the local exponents $h(\vec{x})$ at a given pixel (which in turn gives the fractal components). The most convenient method, from the numerical point of view, is that of the wavelet transform (see $[29,18,13])$. It is based on the convolution of the EM density with an appropriate function $\Psi(\vec{x})$, the wavelet, which is resized using a scale variable $r$ to focus the convenient details at each scale. We thus define the wavelet projection $T_{\Psi}^{r} d \mu(\vec{x})$ at the point $\vec{x}$ and the scale $r$ as

$$
T_{\Psi}^{r} d \mu(\vec{x})=\int d \vec{y}|\nabla C|(\vec{y}) \Psi_{r}(\vec{x}-\vec{y}),
$$

where $\Psi_{r}(\vec{x}) \equiv\left(1 / r^{2}\right) \Psi(\vec{x} / r)$. It can be proven [30] that the EM verifies the multifractal scaling, Eq. (15), if and only if

$$
T_{\Psi}^{r} d \mu(\vec{x})=\bar{\alpha}(\vec{x}) r^{h(\vec{x})}
$$

where $h(\vec{x})$ is the same exponent as in Eq. (15) and $\vec{\alpha}(\vec{x})$ is a suitable function. This multiresolution method allows for a very good discrimination of the sets $F_{h}$; once they are obtained, their irregular (fractal) nature is clear by simple visual inspection.

Applying the theory to the data, using the previously computed values of $\tau_{2}$ and $\beta$ (Fig. 3), it is obtained that the MSM has a dimension $D_{\infty} \approx 1$, which makes plausible that it consists of segments of curves. Visual inspection of this set (Figs. 5 and 6) reveals that it is rather close to the edges present in the chromatic components.
Comparison of the MSM's of the two chromatic systems, RGB and $l \alpha \beta$, shows that they have qualitatively different geometrical contents. Figures 5 and 6 exemplify the question on a representative image.

(i) The RGB system is highly geometrically redundant. Simple visual inspection of the gray-level representations of the three chromatic variables (first row of Fig. 5) shows three very similar scenes. This fact is confirmed by the multiresolution analysis (second row of Fig. 5). To characterize this geometrical redundancy, we measured the relative density of the different MSM's and of their intersection across the whole ensemble. The values obtained are red MSM: 29.95\%; green MSM: 29.89\%; blue MSM: 23.75\%. The relative density of the intersection of the three sets is $19.60 \%$. This means that the intersection contains $65.44 \%$ of the red MSM, $65.57 \%$ of the green MSM, and $82.53 \%$ of the blue MSM, so it is clear that the three MSM's share a significant amount of geometrical content. In other words: the luminosity edges typically occur simultaneously in all three chromatic components in this representation.

(ii) The l $\alpha \beta$ system has significant geometrical differences among its chromatic variables. There are very well defined borders that are shared by all the variables; however, several geometrical structures are apparent only in one of the three chromatic components of the image. It seems that this representation could enhance the separation of different types of objects attending to their color distribution. This result seems very appealing. We computed again the densities of the different sets (each MSM and the intersection of the three) across the whole ensemble of images. The values are the following: $l$ MSM: 40.96\%; $\alpha$ MSM: $45.30 \% ; \beta$ MSM: $44.65 \%$. The set resulting from the intersection of the three has a density of $13.93 \%$, which means that it contains $34.01 \%$ of the $l$ MSM, $30.75 \%$ of the $\alpha$ MSM, and $31.20 \%$ of the $\beta$ MSM. In this sense, these chromatic variables pos- 
$l$

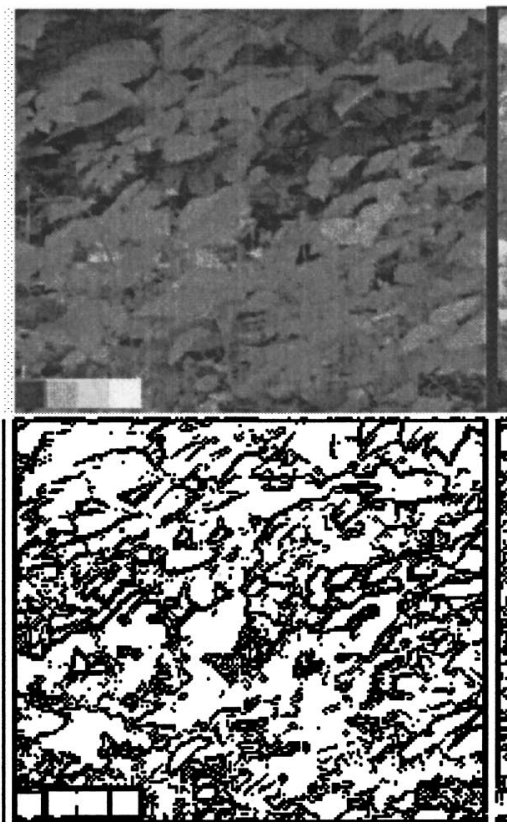

$\alpha$

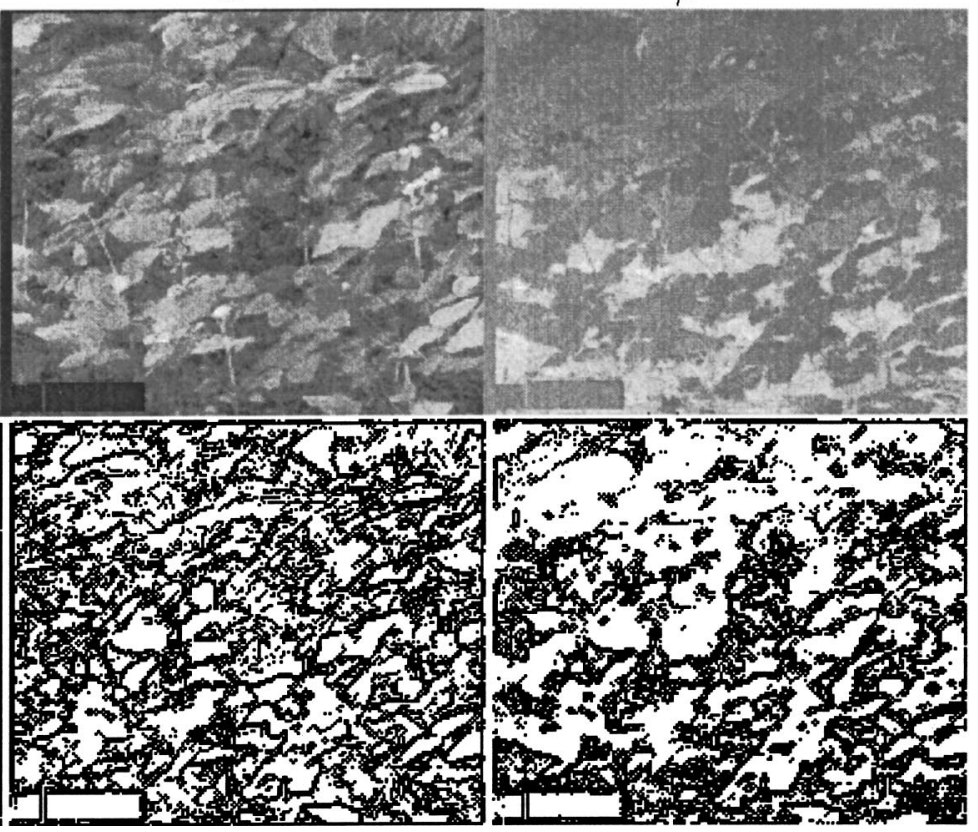

FIG. 6. Gray-level representations of the $l \alpha \beta$ system (top) and the associated MSM's (bottom) of the Park2 image. The MSM's were taken as the sets of points having exponent $h=-0.5 \pm 0.1$. The $\beta$ component appears rather saturated. Although the most important transitions are present in the three chromatic variables, there is a significant amount of structure detected by only one of the three, reducing the geometrical redundancy.

sess less geometrical redundancy than those in the RGB system. This is explained by the fact that there are sharp edges which belong just to one of the spaces and not to the other two, in contrast with the situation of the RGB system.

The inspection of these results reveals two interesting features: first, the MSM's associated to the $l \alpha \beta$ system are denser than those of the RGB system. This is mainly caused by the logarithmic transformation from RGB to $l \alpha \beta$, which increases the contrast on average and so enhances details. Second, for the $l \alpha \beta$ system, the ratio between the number of pixels in the intersection and in each of the MSM's is less than half of the same ratio in the RGB system. This makes more evident the appearance of different geometrical structures. The higher degree of independence in the $l \alpha \beta$ system is rather natural because of their construction: they are decorrelated variables (see [21]).

\section{INFORMATION CONTENT}

The classification of the points in the images according their multifractal structure has revealed significative differences between the RGB and the $l \alpha \beta$ schemes. Although the geometrical coincidences and the separation of features seem very informative, it is convenient to have a more quantitative criterion than the rather coarse density estimation. In particular, it would be desirable to characterize the amount of information conveyed by the MSM's and the degree of redundancy among them. This characterization can be done by using the concepts of entropy and mutual information.

Given a random variable $X$ with a probability distribution $\rho_{X}(x)$, its entropy (or total information) $H_{X}$ is defined as

$$
H_{X}=-\int d x \rho_{X}(x) \log _{b} \rho_{X}(x)
$$

It has no actual units, but depending on the basis $b$ of the logarithm a unit name is usually given. For $b=2$, which we will use, it is expressed in bits. For discrete variables this quantity is always a finite, positive number, which is maximum for uniformly distributed variables. For continuous variables it does not even need to be defined, and can have a positive or negative value. For this reason, when a discretization of a continuous variable is considered, the discretization range is very relevant. It can be proved (see, for instance, [31]) that for discretized variables the entropy represents an optimal bound for the average amount of digits to be used in the encoding of events described by $X$.

Given two random variables, $X$ and $Y$, with marginal probability distributions $\rho_{X}(x)$ and $\rho_{Y}(y)$ and joint probability distribution $\rho_{X Y}(x, y)$, we define the mutual information between $X$ and $Y, I_{X Y}$, as

$$
I_{X Y}=\int d x \int d y \rho_{X Y}(x, y) \log _{b} \frac{\rho_{X Y}(x, y)}{\rho_{X}(x) \rho_{Y}(y)} .
$$

It is expressed in the same units as the entropy. It can be proved that it is always a positive quantity which only vanishes when $\rho_{X Y}(x, y)=\rho_{X}(x) \rho_{Y}(y)$, so in a sense it is a measure of the statistical independence of the variables $X$ and $Y$. In fact, it gives the amount of information shared by the two variables:

$$
I_{X Y}=H_{X}-H_{X \mid Y}=H_{Y}-H_{Y \mid X},
$$

where $H_{X \mid Y}$ is the conditional entropy, defined as

$$
H_{X \mid Y} \equiv \int d y \rho_{Y}(y)\left(-\int d x \rho_{X \mid Y}(x \mid y) \log _{b} \rho_{X \mid Y}(x \mid y)\right),
$$


and $\rho_{X \mid Y}(x \mid y)=\rho_{X Y}(x, y) / \rho_{Y}(y)$ is the distribution of $X$ conditioned by $Y$. The conditional entropy is the average of the entropy of $X$ for fixed values of $Y$. It is the part of the entropy of $X$ which is independent of $Y: 0<H_{X \mid Y} \leqslant H_{X}$ and $H_{X \mid Y}$ $=H_{X}$ only if $X$ is independent of $Y$. Thus, the mutual information, according to Eq. (23), measures the amount of bits of $X$ which can be predicted by the knowledge of $Y$ and vice versa.

The definition of mutual information can be extended to more than two variables, although not in a unique way. We will work with the information shared by three variables $X$, $Y$, and $Z$, which can be expressed as

$$
I_{X Y Z}=I_{X Y}-I_{X Y \mid Z},
$$

where $I_{X Y \mid Z}$ is the averaged mutual information between $X$ and $Y$ for fixed values of $Z$ [that is, it is computed using $\left.\rho_{X Y \mid Z}(x, y \mid z)\right]$. The interpretation of this quantity is similar to that of Eq. (23). The last term is the amount of information between $X$ and $Y$ which is not shared by $Z$, while the difference gives the information shared by the three variables. Contrary to the mutual information of two variables [Eq. (23)], which is always positive, $I_{X Y Z}$ can be negative. This happens when fixing the value of $Z$ causes the relation between $X$ and $Y$ to become less random, increasing their statistical dependence. As an extreme case we consider $X=Y$ $+Z$, with $Z$ independent of $Y$. Fixing $Z$, the quantity $I_{X Y \mid Z}$ takes its maximum value $H_{Y}$, and $I_{X Y Z}=-H_{Y \mid X}$. On the other hand, a positive value of $I_{X Y Z}$ indicates that fixing $Z$ the other two variables become more independent.

It can be proved that

$$
I_{X Y Z}=I_{Y Z}-I_{Y Z \mid X}=I_{X Z}-I_{X Z \mid Y},
$$

that is, the difference between the two mutual informations is independent of which variable is kept fixed. An explicitly symmetric expression is given by

$$
I_{X Y Z}=I_{X Y}+I_{X Z}+I_{Y Z}-K_{X Y Z}
$$

where

$K_{X Y Z}=\int d x \int d y \int d z \rho_{X Y Z}(x, y, z) \log _{b} \frac{\rho_{X Y Z}(x, y, z)}{\rho_{X}(x) \rho_{Y}(y) \rho_{Z}(z)}$.

We now start the information analysis of the multifractal densities $|\nabla C|(\vec{x})$ of the chromatic components, when the point $\vec{x}$ runs across particular geometrical sets. We are interested in measuring entropies and mutual information among the three variables of each chromatic system, at the same pixel $\vec{x}$, averaging over all the pixels of the image ensemble.

For each chromatic system we consider three different geometrical sets. The first is obtained from the whole images; the second contains the pixels common to the MSM's of the three components; and the third is given by the pixels common to the second MSM's. The comparison between these sets will give valuable knowledge about the distribution of information in the image.

We obtained the following results.

(i) RGB system. The results are summarized in Table I. It is observed that the entropic content of the MSM is larger
TABLE I. Entropies and mutual informations in the RGB system, arranged in columns. The first three columns represent the entropies of the red, green, and blue variables. The next three columns represent the mutual information between the different pairs. The last two columns represent the two definitions we have given for the mutual information of the three variables altogether. The rows refer to the spatial extent of the sampling: the whole image (top), the intersection of the three MSM's (middle), and the intersection of the three first manifolds (bottom); here, $h_{1}=-0.3 \pm 0.1$. All the data are expressed in bits. The bit depth of $|\nabla C|(\vec{x})$ for each component was taken to be eight bits, which means that it has been discretized in $2^{8} \equiv 256$ values; thus the maximal possible value of each entropy is 8 .

\begin{tabular}{lcccccccc}
\hline \hline & $H_{\mathrm{R}}$ & $H_{\mathrm{G}}$ & $H_{\mathrm{B}}$ & $I_{\mathrm{GB}}$ & $I_{\mathrm{RB}}$ & $I_{\mathrm{RG}}$ & $K_{\mathrm{RGB}}$ & $I_{\mathrm{RGB}}$ \\
\hline Whole image & 4.77 & 4.78 & 4.13 & 0.94 & 0.90 & 2.76 & 3.76 & 0.84 \\
$F_{\infty}$ & 5.35 & 5.34 & 4.69 & 1.30 & 1.25 & 3.22 & 4.64 & 1.13 \\
$F_{h_{1}}$ & 4.92 & 4.93 & 4.73 & 0.86 & 0.81 & 2.78 & 3.72 & 0.73 \\
\hline \hline
\end{tabular}

than that of the whole image, while for the following manifold this entropic increase is not present. Besides, comparing the entropy of the second MSM with that of the whole image, it is seen that they are similar (again, the lack of contrast in the blue component causes some of the pixels in the MSM to be detected as belonging to the second MSM). It was also observed that less singular fractal components have the same entropy as the whole image. The conclusion that can be extracted from this is that the number of frequent values that $|\nabla C|(\vec{x})$ takes on the MSM is greater than on the whole image. On the other hand, sampling on the second MSM (or in any of the next fractal components) is equivalent to sampling on the complete scene. It is then concluded that this variable distinguishes the MSM from the other fractal components by having a greater number of useful bits on it. This system exhibits a rather large amount of mutual information between pairs of variables, which is maximal for the pair Red-Green (those with the most similar wavelength ranges) for the three geometrical sets. Related to this, one also observes that $I_{\mathrm{GB}} \simeq I_{\mathrm{RB}} \simeq I_{\mathrm{RGB}}$, that is, the information shared by the pairs GB or RB is close to the information common to the three variables. This shows again the strong dependence between the red and green components. Notice that the mutual information also follows the same changes shown by the entropies over the geometrical sets.

(ii) $l \alpha \beta$ system. Table II summarizes the results obtained for this system. We first notice that all the entropies are larger than those of the RGB components. It also exhibits an

TABLE II. Entropies and mutual information for the $l \alpha \beta$ system, arranged in columns. The first three columns represent the entropies for the $l, \alpha$, and $\beta$ variables, and the following are arranged as those of Table I. The rows refer to the same geometrical sets as in Table I. All the data are expressed in bits. The bit depth for each component was taken to be eight bits.

\begin{tabular}{lcccccccc}
\hline \hline & $H_{l}$ & $H_{\alpha}$ & $H_{\beta}$ & $I_{l \alpha}$ & $I_{l \beta}$ & $I_{\alpha \beta}$ & $K_{l \alpha \beta}$ & $I_{l \alpha \beta}$ \\
\hline Whole image & 6.45 & 5.84 & 5.25 & 0.15 & 0.13 & 0.10 & 0.67 & -0.29 \\
$F_{\infty}$ & 6.48 & 6.04 & 5.60 & 0.29 & 0.24 & 0.19 & 1.71 & -0.99 \\
$F_{h_{1}}$ & 6.44 & 5.85 & 5.22 & 0.19 & 0.15 & 0.13 & 0.97 & -0.50 \\
\hline \hline
\end{tabular}


entropic increment of the MSM with respect to the whole images, although it is smaller than for the RGB system. Again the entropies defined over the second MSM are rather similar to those of the whole image. The two-variable mutual information is rather limited, which is expected because of the decorrelation achieved by this system. Contrary to the RGB system, now $I_{l \alpha \beta}<0$, which yields an increase of the mutual information between two variables when the third is known. Let us emphasize that on the pixels common to the three MSM's, the value of $I_{l \alpha \beta}$ is still more negative: this implies that on this set the degree of dependence of the gradients is larger than over the whole image. Given that in this system the three two-variable mutual informations are rather close, the argument applies to the three possible pairs.

\section{CONCLUSIONS}

In this work we have addressed the question of whether color-natural scenes exhibit non-Gaussian statistics related to the gradient of the chromatic components. This was done for each of the three chromatic channels of two different chromatic systems: the cone responses RGB and their decorrelated version $l \alpha \beta$ [21]. The main conclusion is that natural color images exhibit multiscaling effects similar to monochromatic images $[12,13]$, for both chromatic systems. In particular, it has been checked that the multiscaling statistical properties are very well described in the context of multiplicative processes, with just two free parameters.

An explicit decomposition of the images in their fractal components was also done using a wavelet technique. The most important of these components [which we have called the most singular manifold (MSM)] is given by the obvious contours in each chromatic channel. It was found that the $\mathrm{RGB}$ and the $l \alpha \beta$ systems have rather different geometrical structure. While the first exhibits a great deal of redundancy, in the sense that the fractal components of the three channels are quite similar, the decorrelated system extracts different features in the different channels.

In addition to this, this fractal structure helps to detect the most informative pixels in the images. To study this issue, we have computed several information quantities: the total entropy, the mutual information between pairs of channels, and the information shared by the three channels of a given system. This analysis reveals that the MSM contains the most informative pixels in the image, for the six considered channels. There are differences between the RGB and the $l \alpha \beta$ systems. All the measured quantities show that the first is highly redundant, in that a given channel contains a large amount of information about the others. On the contrary, the decorrelated system has eliminated some redundancy. However, there is still a substantial amount of mutual information, which is maximal over the MSM.

The results found in this work also contribute to confirm the robustness of the multiscaling properties of natural images. These properties are not a peculiarity of monochromatic images but they are also present in color images, regardless of the chromatic system used.

We want to emphasize that the multiscaling properties discussed here give a priori information about what a natural image is, reducing the entropy of the ensemble of natural scenes. This could be useful for explaining the processing of color images in the early stages of the visual pathway. The existence of such a rich structure suggests that the secondorder statistics alone do not contain all visually significant information. Instead, as it was already found for gray-scale images [20], color processing filters will be fully defined once the non-Gaussian properties described here are included.

\section{ACKNOWLEDGMENTS}

This work is funded by the Spanish Ministery of Education under Grant No. PB96-0047 and the National Science Foundation under Grant Nos. IBN-9413357 and IBN9724028. A.T. is financially supported by a FPI grant from the Comunidad Autonoma de Madrid. Financial support was also provided by the Alfred P. Sloan Foundation (to D.L.R.).
[1] H. B. Barlow, Possible Principles Underlying the Transformation of Sensory Messages, in Sensory Communication, edited by W. Rosenblith (MIT Press, Cambridge, MA, 1961), p. 217.

[2] S. B. Laughlin, Z. Naturforsch. B 36, 910 (1981).

[3] J. J. Atick, Network 3, 213 (1992).

[4] J. H. van Hateren, J. Comp. Physiol. A 171, 157 (1992).

[5] G. J. Burton and I. R. Moorhead, Appl. Opt. 26, 157 (1987).

[6] D. J. Field, J. Opt. Soc. Am. 4, 2379 (1987).

[7] J. J. Atick, Z. Li, and A. N. Redlich, Neural Comput. 4, 559 (1992).

[8] Zhaoping Li and J. J. Atick, Neural Comput. 6, 127 (1994).

[9] D. J. Field, in Wavelets, Fractals, and Fourier Transforms, edited by M. Farge, J. C. R. Hunt, and J. C. Vassilicos (Clarendon Press, Oxford, 1993), pp. 151-193.

[10] D. Ruderman and W. Bialek, Phys. Rev. Lett. 73, 814 (1994).

[11] D. Ruderman, Network 5, 517 (1994).

[12] A. Turiel, G. Mato, N. Parga, and J.-P. Nadal, Phys. Rev. Lett. 80, 1098 (1998).

[13] A. Turiel and N. Parga, Neural Comput. 12, 763 (2000).
[14] A. Turiel, G. Mato, N. Parga, and J.-P. Nadal, Self-Similarity Properties of Natural Images, Proceedings of NIPS'97 (MIT Press, Cambridge, MA, 1998).

[15] K. Falconer, Fractal Geometry: Mathematical Foundations and Applications (John Wiley and Sons, Chichester, 1990).

[16] G. Parisi and U. Frisch, in Turbulence and Predictability in Geophysical Fluid Dynamics, Proceedings of the International School of Physics "Enrico Fermi," Varenna, 1983, edited by M. Ghil, R. Benzi, and G. Parisi (North-Holland, Amsterdam, 1985), pp. 84-87.

[17] T. Lindberg, Scale Space Theory in Computer Vision (Kluwer Academic, Dordrecht, 1994).

[18] S. Mallat and S. Zhong, in Wavelets and Their Applications, edited by M. B. Ruskai et al. (Jones and Bartlett, Boston, 1991), pp. 67-104.

[19] A. Nevado, A. Turiel, and N. Parga, Network 11, 131 (2000).

[20] A. Turiel and N. Parga (unpublished).

[21] D. Ruderman, T. Cronin, and C. C. Chiao, J. Opt. Soc. Am. A 15, 2036 (1998). 
[22] A. Stockman, D. I. A. MacLeod, and N. E. Johnson, J. Opt. Soc. Am. A 10, 2491 (1993).

[23] G. Wyszecki and W. S. Stiles, Color Sciences: Concepts and Methods, Quantitative Data and Formulae, 2nd ed. (John Wiley \& Sons, New York, 1982).

[24] E. A. Novikov, Phys. Rev. E 50, R3303 (1994).

[25] B. Dubrulle, Phys. Rev. Lett. 73, 959 (1994).

[26] B. Castaing, J. Phys. II 6, 105 (1996).

[27] Z.-S. She and E. Leveque, Phys. Rev. Lett. 72, 336 (1994).
[28] A. Turiel and A. del Pozo (unpublished).

[29] A. Arneodo et al., Ondelettes, Multifractales et Turbulence (Diderot Editeur, Paris, 1995).

[30] A. Arneodo et al., in Wavelets. Theory and Applications, edited by G. Erlebacher, Hussaini M. Yousuff, and L.M. Jameson, ICASE/LaRC Series in Computational Science and Engineering (Oxford University Press, New York, 1996), p. 349.

[31] T. M. Cover and J. A. Thomas, Elements of Information Theory (John Wiley \& Sons, New York, 1991). 